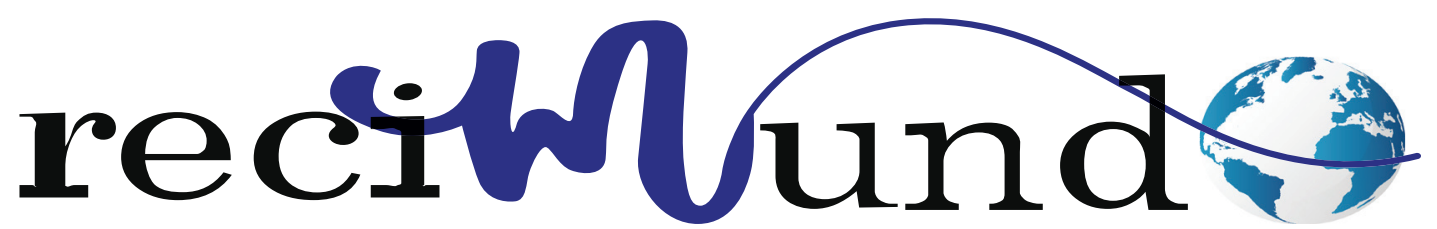

Revista Científica Mundo de la Investigación y el Conocimiento

DOI: $10.26820 /$ recimundo/5.(1).enero.2021.164-174

URL: http://recimundo.com/index.php/es/article/view/995

EDITORIAL: Saberes del Conocimiento

REVISTA: RECIMUNDO

ISSN: 2588-073X TIPO DE INVESTIGACIÓN: Artículo de Revisión PAGINAS: $164-174$

\title{
Liderazgo del siglo XXI en las instituciones públicas
}

21st century leadership in public institutions

Liderança do século 21 nas instituições públicas

Cristina Guadalupe Ordóñez Espinoza'; María Carlina Ordóñez Espinoza²; Renán Teodoro Rodríguez Pillaga3; Mariory Prisila Orbe Guaraca ${ }^{4}$

RECIBIDO: 28/11/2020 ACEPTADO: 06/12/2020 PUBLICADO: 31/01/2021

1. Magister en Gestión Empresarial; Diploma Superior en Educación Universitaria por Competencias; Ingeniera Empresarial; Docente de la Unidad Académica de Administración de Empresas de la Universidad Católica de Cuenca, Extensión Cañar, Ecuador; cgordoneze@ucacue.edu.ec; (iD https://orcid.org/0000-0001-5781-7781

2. Magister en Intervención y Educación Inicial; Diploma Superior en Educación Universitaria por Competencias; Licenciada en Ciencias de la Educación Especialidad Psicología Educativa y Orientación Vocacional; Profesora de Segunda Enseñanza en la Especialidad de Psicología Educativa y Orientación Vocacional; Técnico Superior en Procesamiento Analista en Sistemas de Computación; Docente de la Unidad Educativa Remigio Crespo Toral; Ecuador; karly_ordez@yahoo.es; in https://orcid.org/0000-0003-0317-2484

3. Magister en Gestión Empresarial; Especialista en Docencia Universitaria; Diploma Superior en Tributación; Ingeniero Empresarial; Director de Carrera de Administración de Empresas de la Universidad Católica de Cuenca, Extensión Cañar; Ecuador; rtrodriguezp@ucacue.edu.ec (D) https://orcid.org/0000-0002-3740-6755

4. Magister en Gestión Empresaria; Ingeniera Empresarial; Técnico Superior en Secretariado Ejecutivo Bilingüe; Docente de la Unidad Académica de Administración de Empresas de la Universidad Católica de Cuenca, Extensión Cañar; Ecuador; mporbeg@ucacue.edu.ec (iD https://orcid.org/0000-0002-4239-5675

CORRESPONDENCIA

Cristina Guadalupe Ordóñez Espinoza

cgordoneze@ucacue.edu.ec

Cañar, Ecuador

() RECIMUNDO; Editorial Saberes del Conocimiento, 2021 


\section{RESUMEN}

La investigación en el ámbito educativo entorno a los efectos del liderazgo sobre el hecho educativo, ha evolucionado hacia una visión que enfoca los contextos en los que los líderes trabaja. Hoy en día, una gran proporción de jóvenes abandonan la escuela porque ven la educación formal como irrelevante y se vuelven apático. El presente estudio de tipo investigación documental, analiza prácticas fundamentales de un buen liderazgo, para mejorar la calidad de la enseñanza y el aprendizaje; tales como rediseño de la organización, desarrollo personal, estrategias de motivación, en fin, desarrollo de estrategias de desempeño profesoral que inciden directamente en el rendimiento escolar; asi como estrategias para impulsar liderazgos exitosos en el alumnado, estudio este, que procura impulsar futuras investigaciones y constituirse en documento formativo para los lideres que requiere la sociedad en el siglo XXI.

Palabras clave: Líder, liderazgo, liderazgo escolar, educación del siglo XXI, desempeño laboral.

\section{ABSTRACT}

Research in the field of education on the effects of leadership on education has evolved towards a vision that focuses on the contexts in which leaders work. Today, a large proportion of young people drop out of school because they see formal education as irrelevant and become apathetic. This documentary research study analyzes fundamental practices of good leadership to improve the quality of teaching and learning, such as organizational redesign, personal development, motivational strategies, in short, the development of teacher performance strategies that have a direct impact on school performance, as well as strategies to promote successful leadership in students. This study seeks to promote future research and become a formative document for the leaders that society requires in the 21 st century.

Keywords: Leader, leadership, school leadership, 21st century education, job performance.

\section{RESUMO}

A pesquisa no campo da educação sobre os efeitos da liderança na educação evoluiu para uma visão que se concentra nos contextos em que os líderes trabalham. Hoje, uma grande proporção de jovens desiste da escola porque vê a educação formal como irrelevante e se torna apática. O presente estudo de tipo pesquisa documental, analisa práticas fundamentais de uma boa liderança, para melhorar a qualidade do ensino e da aprendizagem; tais como redesenho da organização, desenvolvimento pessoal, estratégias de motivação, em suma, desenvolvimento de estratégias de desempenho dos professores que afetam diretamente o desempenho escolar; assim como estratégias para promover uma liderança bem sucedida nos estudantes, este estudo, que procura promover pesquisas futuras e tornar-se um documento formativo para os líderes que a sociedade exige no século XXI.

Palavras-chave: Líder, liderança, liderança escolar, educação do século 21, desempenho profissional. 


\section{Introducción}

El siglo XXI caracterizado por los vertiginosos cambios tecnológicos, la globalización, y la transculturización, ha significado grandes desafíos para la educación, que demanda cada día con mayor ahínco, la adquisición de habilidades, capacidades y conocimientos requeridos para sobrevivir y triunfar en el mundo del hoy. La preparación de los individuos para el trabajo y para dichos retos, ha implicado que la educación media se vincule al mercado de trabajo, adoptando preceptos en la enseñanza, tales como la calidad, eficiencia, competitividad, excelencia, y el liderazgos como elementos fundamentales para alcanzar el desarrollo cognitivo del individuo.

La investigación en el ámbito educativo entorno a los efectos del liderazgo sobre el hecho educativo, ha evolucionado hacia una visión que enfoca los contextos en los que los líderes trabajan, cómo se desempeñan, como responden en determinadas situaciones, en fin determinar modelos de liderazgos que permitan la enseñanza y la orientación de los mismos. Luego, descubrir cómo esos modelos tienen influencia en el desempeño estudiantil y en el aprendizaje, es de vital importancia para la pedagogía del mundo de hoy. De tal forma que las investigaciones se orientan hacia identificar como líderes exitosos crean condiciones en sus escuelas que promueven el aprendizaje de los estudiantes (HALLINGER \& HECK, 1996) ; Asi como la identificación de cómo lo distintos tipos del liderazgo explican la variación en el rendimiento de los estudiantes; por ultimo la importancia de la apropiación por parte de estos de la misión y las metas de la escuela, la cultura, la participación en la toma de decisiones y relaciones con los padres y la comunidad en general.

Estas son variables fundamentales sobre la potencial influencia de lo líderes éxitosos en la educación y en el desempeño de los estudiantes fuera de a escuela. Este es el principal objetivo de la investigación en curso.

Hoy en día, una gran proporción de jóvenes abandonan la escuela porque ven la educación formal como irrelevante y se vuelven apático y desconectado de la educación formal. Una creciente desilusión del valor agregado que proporciona la educación como vehículo para la integración social y el desempeño en el mercado laboral. Hoy muchos jóvenes cuestionan el "retorno de la inversión" de rutas educativas tradicionales de "alto estatus" (UNESCO, 2015). Si bien muchos factores contribuyen a la desconexión, existe la creencia general de que secundaria. Las tasas de deserción de estudiantes reflejan una incapacidad por parte de los sistemas educativos para vincular el contenido académico con el mundo real experiencia.

En la presente investigación se indagara sobre algún modelo de liderazgo, basado en la experiencia de los profesores y de los profesionales en ejercicio, para determinar cuales son los aprendizajes esenciales en cada uno de los niveles.

\section{Método}

El presente estudio es de tipo investigación documental, revisión bibliográfica, en medios electrónicos, artículos científicos y demás fuentes hemerográficas permite identifica las diversas posturas en torno a la importancia que reviste el liderazgo en la educación pública, en pleno siglo XXI. Se indaga sobre los distintos modelos de liderazgo, el impacto que tiene en la enseñanza, a través de la revisión de investigaciones de campo, así como de análisis de las reformas educativas orientadas en este sentido, en países de América latina.

\section{Definiendo Liderazgo}

Existen dos ejes sobre los que se centra el liderazgo el primero de ello asociado a la " 
direccionalidad", basado en la puta establecida por el líder y la segunda se atribuye a "marcar influencia", distintos autores señalan los modos de ejercer el liderazgo, uno de ellos, es el modelos indicado por (Yukl, 1994, pág. 4), el liderazgo incide en

“.. la elección de objetivos para el grupo u organización, la organización de actividades laborales para lograr objetivos, la motivación de los seguidores para lograr los objetivos, el mantenimiento de relaciones de cooperación y trabajo en equipo y la obtención de apoyo y cooperación de personas ajenas al grupo u organización ".

Respecto al liderazgo y su capacidad de ejercer influencia sobre otras personas, éste puede originarse de asumir las líneas propuestas como premisa para una acción (Bolívar, 2010) y se fundamenta sobre el arte al influir sobre personas, y la aplicación de determinadas acciones en búsqueda de cubrir con las expectativas del líder, que a su vez significa cumplir con las expectativas de la organización.

En definitiva, consiste en que los individuos a través de sus habilidades ejecuten un trabajo desde su motivación, la disciplina y estructura para cumplir con los objetivos de un entorno dado, donde se ubican. (Semprún-Perich \& Fuenmayor-Romero, 2007)

Mienstra que desde la perspectiva de la Psicología social, la Dra (Mc Guire, 2020) plantea que el liderazgo se expresa en el rol de la personalidad de los seres humanos en el análisis de grupos pequeños. Y en la sociología, se muestra como la influencia que puede ejercer éste sobre la colectividad.

Por su parte Guillen \& Guil (2001, p. 299) indica que:

"Es la influencia que ejerce un individuo sobre un grupo, y por líder, la persona con capacidad de ejercer tal influencia, independiente de los motivos que permiten a tal persona acceder al ejercicio de dicha influencia".

En tal sentido, el líder debe desarrollar una capacidad de adaptabilidad de forma rápida, así como "una lectura avanzada de lo que la organización necesita según las exigencias del entorno" (Mc Guire, 2020); a tal efecto, las personas que le rodean al dejarse llevar por esa influencia, generan una relación y comunicación con dicho líder, construyendo su propia personalidad con características que le permitan potenciar esa motivación, y mantener la disciplina del equipo para alcanzar la meta prevista por la misma organización.

Sin embargo, cabe mencionar el líder tienen sus propias motivaciones, aún y cuando algunas conjuguen y coincidan con la de la organización. Planifica los métodos para desarrollar tareas, y las que cada persona del equipo realizará. En consecuencia, las personas participan desde la motivación del líder, en conjunto con las motivaciones de la organización. (Mc Guire, 2020)

De tal forma que las motivaciones pueden llegar a su máxima expresión una vez terminada la meta, al menos que el líder aproveche el conocimiento, y habilidades aprendidas en el trabajo de equipo y pueda seguir operando, esto atribuido a la transformación interna que en el proceso vive el individuo. Mientras que si los principios no fueran los mismos, se encontraría ante la dicotomía, de efectuar tareas que no le generan sentido y que pueden por el contrario perjudicar dicha transformación individual dentro del colectivo.

En importante acotar que el liderazgo en si mismo evoluciona al igual que lo hacen las personas y las exigencias a las que están sometidas, de tal forma que quien ejerce el liderazgo no debe perder de vista lo funda-

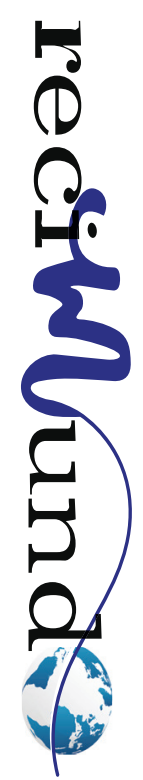


mental de provocar un transformación interna en quienes influencia para el desarrollo evolutivo y de crecimiento personal, que les permita la adaptabilidad a los cambios, sino la monotonía ante tantos elementos cambiantes a o externo le conllevan al fracaso de la organización.

En consecuencia se puede decir que el liderazgo implica procesos que lleven a cumplir las metas como:

1. Influenciar

2. Toma de decisiones,

3. Resolución de conflictos

4. Comunicación efectiva

5. Regular emociones, entre otras. (Mc Guire, 2020)

En síntesis una persona debe organizar, dirigir y planear el propio trabajo y el ajeno, tomando decisiones, aplicando normas y diseñando soluciones; su vez de ser reconocido por otros por ser y ejercer su liderazgo.

\section{Efectos de los líderes en el aprendizaje}

Los efectos de los líderes en el aprendizaje puede ser comprendido en base a los líderes escolares y los efectos indirectos que este liderazgo escolar causa sobre el aprendizaje de los estudiantes, a continuación tres tipos diferentes de investigación, confirman y especifican alguno de estos, así se tienen:

- En una investigación cualitativa (Gezi, 1990) desarrolla estudio de caso que en entornos escolares excepcionales, plantea como conclusión que estos son entornos que se cree que contribuyen al aprendizaje significativamente por encima o por debajo de las expectativas. Dicha investigación, basada en Diseños "atípicos", por lo general producen grandes efectos de liderazgo no sólo en los estudiantes y su aprendizaje, pero también en una variedad de condiciones escolares que predisponen condiciones favorables al aprendizaje.

- Una segunda fuente de evidencia de investigación sobre los efectos del liderazgo es a gran escala, en estudios cuantitativos, donde como Evidencia de este tipo reportada entre 1980 y 1998 han sido revisado en varios artículos por (HALLINGER \& HECK, 1996). Estas revisiones concluyen que los efectos directos e indirectos combinados del liderazgo escolar en los resultados de los alumnos es pequeño pero significativo desde el punto de vista educativo.

- El tercer tipo de investigación sobre los efectos del liderazgo es, como el segundo tipo, también a gran escala y de naturaleza cuantitativa. Pero en lugar de examinar en general efectos de liderazgo, estos estudios indagan acerca de los efectos de liderazgo específico, mediante prácticas. La evidencia de este tipo se puede encontrar en , su estudio identificó 21 "responsabilidades" de liderazgo y calcula una correlación promedio entre cada responsabilidad y las medidas de rendimiento estudiantil, los investigadores calcularon un 10 por ciento aumento en los puntajes de los exámenes de los estudiantes de un director promedio que mejoró su "Habilidades demostradas en las 21 responsabilidades por una desviación estándar"

Si bien el análisis de (Waters, Marzano, \& McNulty, 2003) produjo interesantes datos, extrapolaciones de sus estimaciones a los principales efectos sobre los estudiantes el análisis en línea general debe realizarse considerando múltiples elementos y la complejidad de la realidad.

Por su parte, las dos primeras investigaciones contraponen su postura sobre los efectos del liderazgo, ya que sugieren efectos de diferentes magnitudes; pequeño pero significativo en el primer caso y grande en comparación del estándar en el segundo. 
De cualquier forma, los estudios cuantitativos pueden parecerles a los formuladores de políticas fuentes más confiables de evidencia sobre los efectos del liderazgo, tales estudios subestiman sistemáticamente efectos de liderazgo en las escuelas donde es probable que sea de mayor valor y de allí la importancia de identificar cualitativamente y de forma detallada el liderazgo, sus cualidades, su efecto en el rendimiento del aprendizaje y finalmente, precisar los modos de impulsar un liderazgo estudiantil exitoso, así como el ejercicio de los líderes educativos en los recintos escolares y sus formas de alcanzar las metas, a través de estrategias para tales fines.

\section{Los fundamentos del liderazgo exitoso}

Gran parte del éxito de los líderes escolares apuntan a tres amplias categorías sobre el éxito, independientes del contexto en el que se encuentre el líder. Tales prácticas son los fundamentos de un buen liderazgo, y son planteados por diversos autores bajo concepciones diversas pero no antagónicas, así se encuentran:

(HALLINGER \& HECK, 1996) clasifican estas categorías como:
a) Propósitos
b) Personas
c) Estructuras

Conger y Kanungo (1998) refieren a las estrategias:
a) Estrategias de visión
b) Estrategias de creación de eficacia
c) Estrategias de cambio de contexto

Las categorías de (Leithwood K. , 2004) son:
a) Direccionalidad
b) Desarrollo personal
c) Rediseño de organización.

Estas categorías de prácticas de liderazgo según Bass (1997) afirma que han demostrado ser útil en muchos contextos cultura- les y organizativos diferentes, y describe los siguientes elementos como:

- Transformacional El enfoque ha demostrado ser útil para las organizaciones educativas (en estudios de Geijsel, Sleegers, Leithwood y Jantzi, 2003; Yu, Leithwoody Jantzi, 2002; Southworth, 1998; y Mullin y Keedy, 1998), por el éxito de algunos esfuerzos de reforma a gran escala en las escuelas.

- Establecer direcciones, Un aspecto crítico del liderazgo es ayudar a un grupo a desarrollar conocimientos sobre la organización y sus actividades y objetivos que pueden sustentar un sentido de propósito o visión (HALLINGER \& HECK, 1996)

- Explicaciones teóricas fundamentales de la importancia de la dirección de los líderes, son teorías de la motivación humana basadas en objetivos, según dicha teoría, las personas están motivadas por objetivos que encuentran personalmente convincentes, así como tan desafiante pero alcanzable. Tener tales objetivos ayuda a las personas a entender su trabajo y les permite encontrar un sentido de identidad por sí mismos dentro de su contexto de trabajo.

- Desarrollo personal, si bien las direcciones organizacionales claras y convincentes contribuyen significativamente a las motivaciones laborales de los miembros, no son las únicas condiciones para hacerlo. Las capacidades y las motivaciones están influenciadas por la experiencia directa de los miembros de la organización, y esto tiene relación directa con aquellos en roles de liderazgo (Lord y Maher, 1993), así como con el contexto organizacional dentro del cual las personas se desenvuelven (Rowan, 1996).

La capacidad para impulsar y ayudar al desarrollo de las personas depende, en gran parte, del conocimiento que tengan los líderes del "núcleo técnico" de la educación: lo

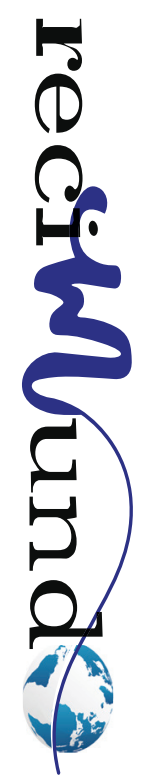


que se requiere para mejorar la calidad de la enseñanza y el aprendizaje, es lo que algunos autores han Ilamado "Liderazgo educativo", habilidad también conocida como inteligencia emocional de los líderes (Goleman, Boyatzis, \& McKee, 2005). Por su parte, la inteligencia emocional se muestra, a través de la atención personal de un líder a un empleado y su habilidad para utilizar las propias capacidades del empleado, en motivar y disminuir la frustración, lo cual transmite un sentido de misión e indirectamente esto aumentará el rendimiento (McColl-Kennedy \& Anderson, 2002).

Prácticas de liderazgo más específicas que ayudan de manera significativa y positiva a desarrollar a las personas incluyen ofrecer estimulación intelectual, proporcionando apoyo y un modelo adecuado.

- Rediseño de la organización, los líderes educativos exitosos desarrollan sus escuelas como organizaciones que apoyan y mantienen el desempeño de los administradores, profesores y estudiantes, mediante de prácticas de liderazgo que contribuyen al desempeño de lo trabajadores y al mejor aprendizaje de los estudiantes, dichas prácticas basadas en culturas y estructuras organizacionales, en donde los miembros participan en la construcción de procesos colaborativos y del desarrollo organizacional de sus escuelas.

- El liderazgo educativo, establece con la dinámica de la acción formativa de las personas fundamentada en ideales filosóficos, por consiguiente, en la formación integral, en cuanto a lo estratégico y técnico con esencia sostenible frente a la comprensión, intervención y trasformación de la cultura, lo ambiental y lo social, impulsando la proyección del ser, hacia el sentido de la educación humana, en lo profesional y personal, sustentado sobre la ética y en la valoración de los recursos de las generaciones futuras.
Es así como (Hargreaves, 2009) fundamenta el desarrollo humano integral, planteando que mas allá de la eficacia competitiva de algunas reformas en la educación, los cambios que se requieren para el aprendizaje de los estudiantes de hoy son procesos sostenibles, democráticos y justos. Señala, que el liderazgo y la mejora educativa sostenibles preservan y desarrollan el aprendizaje profundo de todo aquello que se difunde y que consigue perdurar en el tiempo, y que propicie beneficio para las personas alrededor, en el presente y en el futuro

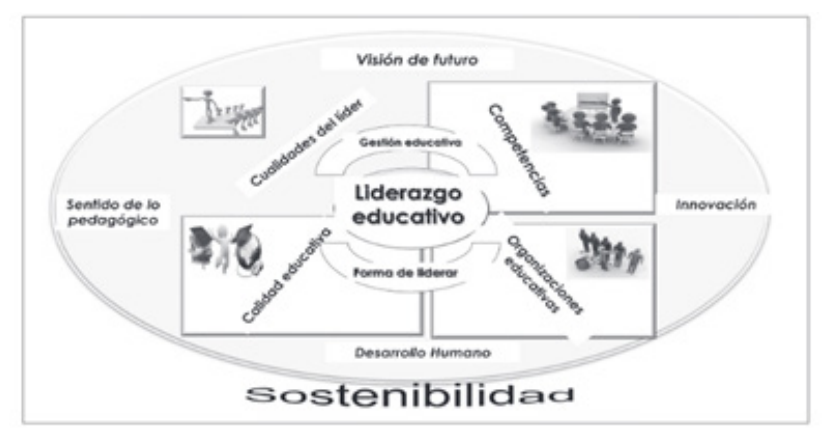

Figura 1. Liderazgo educativo y sostenibilidad

Fuente: (HARGREAVES \& FINK, 2006)

\section{Impacto del liderazgo escolar en el rendi- miento}

En estudio, realizado por (Day, y otros, 2009) al respecto del impacto del liderazgo en los resultados de los alumnos, en torno a 3 categorias, a saber: cognitivos, afectivos y conducta social, llegan a resultados en donde dependen, como variable mediadora, de las condiciones del trabajo docente, cuyo impacto en el aprendizaje se verá moderado por otras variables como el capital cultural de la familia o el contexto organizativo. 


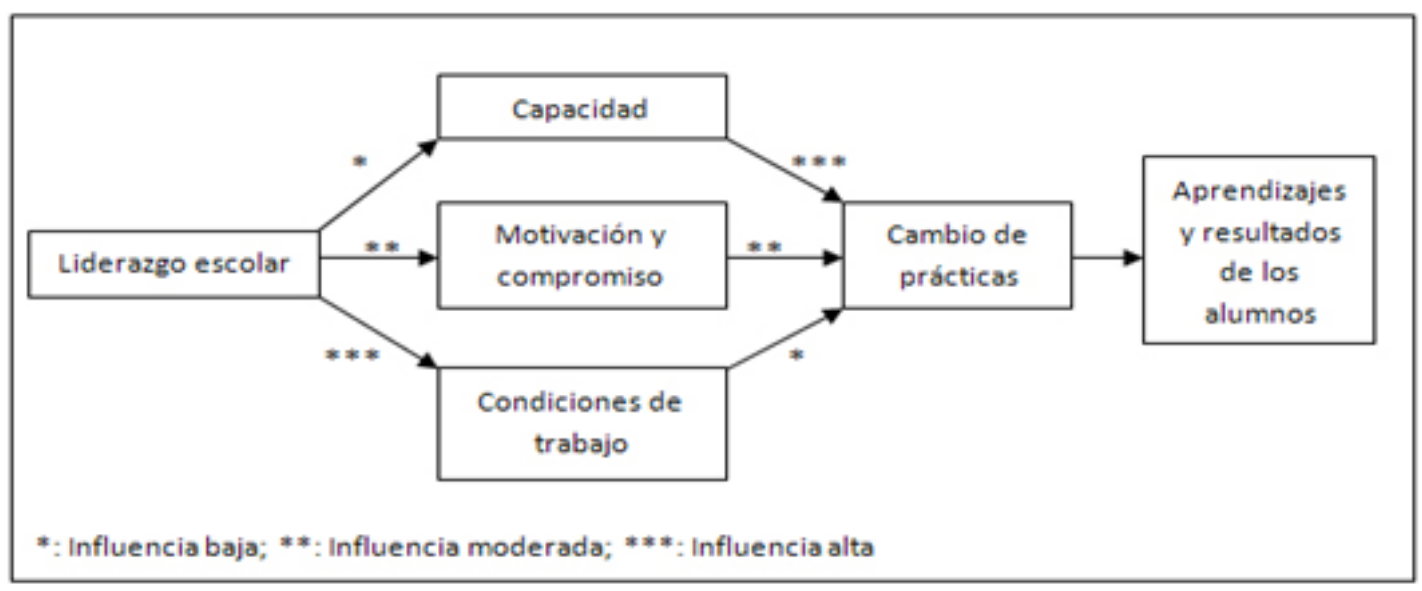

Figura 2. Los efectos del liderazgo escolar

Fuente: (Day, y otros, 2009)

Como indica la Figura 2, los autores plantean una relación directa entre el aprendizaje y el desempeño del profesorado. Sobre el desempeño laboral de los profesores, atribuyen el mismo a la motivación, a su propias capacidades y las condiciones en las que trabajan. En esta investigación de campo, plantean sobre la fuerza de las relaciones : baja, moderada o alta influencia, de acuerdo con los resultados de la investigación. Cuando los directores ejercían este tipo de liderazgo mayor influencia tenían en estas variables intermedias del profesorado que, a su vez, condicionan las nuevas prácticas docentes y, finalmente, los resultados de los alumnos. La Figura 2 muestra, asimismo, que las capacidades docentes son las que mayor influyen en las prácticas, si bien el grado de intervención del director en ellas es menor, no obstante el sentido de eficacia se constituye en una variable de primer orden. Por tanto, los líderes escolares con éxito mejoran la enseñanza y el aprendizaje y, aun y cuando sea de manera indirecta.

De allí que los resultados de los alumnos penda principalmente de la influencia en la motivación del personal, el compromiso, las prácticas de enseñanza y las capacidades del profesorado para el desarrollo del liderazgo. (Day, y otros, 2009)

\section{EI Liderazgo educativo en el siglo XXI}

El liderazgo educativo es establecido desde la acción formativa de las personas, fundamentada en ideales filosóficos, y en la formación integral, donde lo estratégico y técnico hace frente a la comprensión, intervención, transformación de la cultura, lo ambiental y lo social (Sierra Villamil, 2016). El liderazgo define la gestión escolar, en la acción del líder de influir en los demás para que traten de alcanzar los objetivos en la institución escolar (Garbanzo Vargas \& Orozco Delgado, 2010)

Este tipo de liderazgo tiene como resultado el aprendizaje, permitiendo la proyección del Ser hacia el sentido de la educación humana que garantice mejorar la calidad de vida, en lo profesional y personal del individuo. Por tal motivo debe ser aplicado un liderazgo para la mejora continua y sostenible en el desarrollo del aprendizaje profundo que perdure en el tiempo, que se fije en el estudiantado, en pro de su propio benefi-

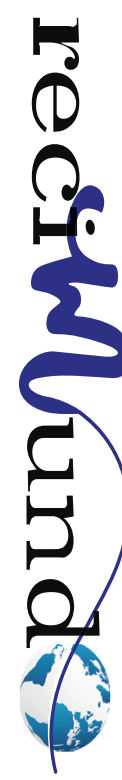


cio, para lo cual debe tomar en cuenta sus historias, modos de vida y los entornos en los que están inmersos (Sierra, 2016).

En otras palabras, los líderes educativos son el profesor como líder dentro del aula, así como el Director de la organización, ya que en ambas se ejerce influencia sobre un grupo de personas para que lleguen a objetivos planteados.

Este liderazgo guarda relación con la influencia de sus propias capacidades, para manejar la información, empoderar y delegar, desde la proyección de su ética y sus valores. Con un sentido fundamentado hacia la claridad de los objetivos que requiere la organización, ofreciendo un valor diferenciador, en las estrategias que domina para implementar proyectos y establecer dinámicas internas de la institución. A su capacidad de abordar y analizar los problemas, crear escenarios de comunicación, de escucha y permitir vías para relacionarse sistemáticamente con la autoridad que le ofrece el cargo (Garbanzo Vargas \& Orozco Delgado, 2010) Un liderazgo que hace énfasis en la toma de decisiones, fundamentada en la colaboración y el conocimiento actualizado de los temas que requiere para cumplir con los objetivos.

Es importante destacar de la investigación realizada por (Day, y otros, 2009) dos tipos de características que garantizan la efectividad del líder educativo, estas pueden ser clasificadas como:

\section{a) Características cognitivas \\ b) Características afectivas}

a) Características cognitivas del líder educativo efectivo: La evidencia revisada contribuyen a los temas de la inteligencia general y otros aspectos del funcionamiento intelectual, las habilidades de resolución de problemas, el conocimiento, la enseñanza y el aprendizaje para un liderazgo exitoso en las escuelas.
Los autores (Day, y otros, 2009) mencionan algunas funciones cognitivas que posee como características un líder efectivo, entre ellas están:

- Inteligencia y otras funciones intelectuales: Donde se asocia la efectividad del líder con el pensamiento creativo y divergente, la habilidad meta cognitiva, la flexibilidad cognitiva.

- Resolución de problemas: Donde se evidencia la experiencia asociada con resolución de problemas efectivo y eficiente dentro de un dominio particular de actividad.

- Conocimiento: Comprenden lo que implica estar inmersos en reformas que tienen alto contenido a dominar. Como nuevos contenidos de planes de estudios y la forma en que se implementarán pedagógicamente. También conocimiento del contenido de liderazgo que lleve a la innovación educativa, tanto como conocimiento de los procesos de aprendizaje para el éxito de resultados de los estudiantes.

b) Características afectivas de líderes efectivos:

Los mismos autores mencionan características afectivas que predominan en las investigaciones sobre líderes educativos efectivos, tales como:

- Personalidad: estable emocionalmente, extrovertido (sociabilidad, sociable, enérgico, hablador, activo), agradable (cortés, flexible, confiado, bondadoso, cooperativo, de buen corazón, tolerante), Consciente (trabajador, orientado al logro, perseverante), abierto a la experiencia (imaginativa, culta, curiosa, original, de mente amplia, inteligente)

- Motivación: Con fuertes necesidades de logro, que les apasiona su trabajo, muy comprometidos emocionalmente y muy motivados. En un estudio, se percibió que muchos tenían altos niveles de energía que motivarían a otros, además 
de ser determinados, persistentes, trabajadores y resistentes (Leithwood, Day, Sammons, \& Harris, 2006).

\section{Conclusión}

La sociedad cambia y se transforma, el crecimiento económico, científico y tecnológico se convierten en indicadores que invitan a repensar la educación del siglo XXI, de tal forma que la construcción de una sociedad que se adapte a dichos cambios, pasa por mejorar la calidad de nuestra educación desde las escuelas. La educación del siglo XXI se encuentra en una encrucijada; por un lado, se busca ganar egresados para incorporarse en forma efectiva al mundo del trabajo, y por el otro, formar ciudadanos disciplinados para responder a las nuevas relaciones que impone la sociedad del conocimiento.

Por lo tanto, se requiere responder al sentido de la educación en cuanto al qué, al para qué, al porqué, al cómo y al hacia dónde. Estos interrogantes definen una propuesta innovadora, que es consecuente con las necesidades del contexto. Es necesario preparara los jóvenes más que para la vida.

Ante lo analizado en el estudio se evidencia la importancia que reviste el impulso de los liderazgos escolares, ganar a los estudiantes para sus proceso de aprendizaje, desde practicas ejemplares que le llamen e incentiven su motivación al aprendizaje es fundamental; así como garantizar que se apropien de los conceptos propios de los líderes que requiere la sociedad hoy en día.

A tal efecto, las estrategias vislumbradas en este análisis, pretende fomentar investigaciones futuras sobre guías, manuales, y en definitiva de carácter formativo para la mejora del desempeño en los profesores en torno al liderazgo que modelan frente al alumnado.
Las potencialidades y oportunidades que trae consigo el siglo XXI y todos los cambios tecnológicos, desarrollo de medios y herramientas, constituyen la posibilidad de reafirmar y sustanciar el rol de la educación en su capacidad para preparar y dotar a los jóvenes para estar en capacidad de abordar la complejidad social, económica y cuestiones ambientales y llevarle al desarrollo científico y al aprovechamiento de sus propias capacidades en este proceso, que le permite interactuar de forma apropiada con el entorno.

\section{Bibliografía}

Beatriz , P., Deborah , N., \& Hunter , M. (2008). Improving School Leadership (Vol. 1). OCDE.

Bolívar, A. (Diciembre de 2010). EL LIDERAZGO EDUCATIVO Y SU PAPEL EN LA MEJORA: UNA REVISIÓN ACTUAL DE SUS POSIBILIDADES Y LIMITACIONES. Psicoperspectivas, Individuo y Sociedad, 9(2).

Day, C., Sammons, P., Hopkins, D., Harris, A., Leithwood, K., Gu, Q., ... Kington, A. (2009). The Impact of School Leadership on Pupil Outcomes. Nottingham: University of Nottingham.

Garbanzo Vargas, G. M., \& Orozco Delgado, V. H. (2010). Liderazgos para una Gestión Moderna de Procesos Educativos. Educación, 34, 15-29.

Gaspar, A., \& Rojas, F. (2006). Bases del liderazgo en educación (Vol. 1). Santiago de Chile, Chile: UNESCO.

Goleman, Boyatzis, \& McKee. (2005). Modelo de liderazgo Primal. University of cambridge.

HALLINGER, P. H., \& HECK, R. H. (1996). Reassessing the principal's role in school effectiveness: A review of empirical research 1980-1995. Educational Administration Quarterly, 32(1), 5-44.

Hargreaves, A. (junio de 2009). El liderazgo sustentable y el cambio en tiempos de confusión. Revista de Educación, 19, 181-195.

HARGREAVES, A., \& FINK, D. (2006). Sustainable Leadership.

Leithwood, K. (2004). Educational Leadership. Temple University Center for Research in Human Development and Education.

Leithwood, K., Seashore Louis, K., \& Stephen, A. (2004). How leadership influences student lear-

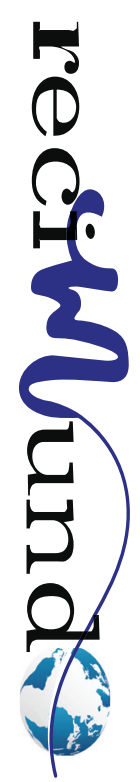


ning. Minnesota: The Wallace Foundation.

Mc Guire, J. (2020). Aspectos de la motivación de las personas como respuesta del liderazgo desde la perspectiva de Neurociencias cognitivas. Obtenido de https://drajolie.cl/2020/03/05/neuroliderazgo-liderazgo-neurocienciascognitivas/

McColl-Kennedy, J. R., \& Anderson, R. D. (2002). Impact of leadership style and emotions on subordinate performance. The Leadership Quarterly, 545-559.

Osandón, L., Caro, M., Magendzo, A., Abraham, M., Lavín, S., González, F., \& Cabaluz, J. F. (julio de 2018). Estado, mercado y currículum escolar: la experiencia chilena (1964-2018). Cuestiones fundamentales y actuales del currículo, el aprendizaje y la evaluación(20). Oficina Internacional de Educación de la UNESCO.

PRÁCTICAS DE LIDERAZGO DIRECTIVO Y RESULTADOS DE APRENDIZAJE HACIA CONCEPTOS CAPACES DE GUIAR LA INVESTIGACIÓN EMPÍRICA (Vol. 7). (2009). Madrid.

Propuesta de la UNESCO para garantizar la educación online durante la pandemia. (2020). Obtenido de Educa web.

Salas Luévano, M. d., Salas Luévano, M. A., \& Herrera Guzmán, B. (s.f.). IMPORTANCIA DEL LIDERAZGO ESCOLAR PARA LA CONSECUCIÓN DE LA CALIDAD EN INSTITUCIONES DE EDUCACIÓN MEDIA SUPERIOR. Recuperado el 1 de 12 de 2020, de http://online.aliat.edu.mx: http://online.aliat.edu.mx/adistancia/Liderazgo/img/lecturas/Importancia_liderazgo_\%20escolar.pdf
Scott, C. L. (2015). WHY MUST LEARNING CONTENT AND METHODS CHANGE IN THE 21st CENTURY? EDUCATION RESEARCH AND FORESIGHT.

Semprún-Perich, R. D., \& Fuenmayor-Romero, J. C. (2007). UN GENUINO ESTILO DE LIDERAZGO EDUCATIVO: ¿UNA REALIDAD O UNA FICCIÓN INSTITUCIONAL? Laurus, 13(23), 350-380.

Sierra Villamil, G. M. (Agosto de 2016). Liderazgo educativo en el siglo XXI desde el emprendimiento sostenible. Escuela Administración y Negocio(81), 111-128.

Tardif, J. (2006). UN pasaje obligé dans la planification de l'évaluation des compétences: déterminer des indicateurs progressifs et terminaux de développement (2a partie). Pédagodie collégiale, 18(2), 13-20.

UNESCO. (2 de Septiembre de 2013). Universalidad de Internet: un medio para crear sociedades del conocimiento y la agenda de desarrollo sostenible después de 2015.

UNESCO. (2015). Replantear la educación:Hacia un bien común Mundial? Paris: UNESCO.

United Nations. (2020). Policy Brief: Education during COVID-19 and beyond. United Nations.

Waters, T., Marzano, R. J., \& McNulty, B. (2003). Balanced Leadership: What 30 about the effect of leadership on student achievement. Denver: McRel.

Yukl, G. (1994). Leadership in Organizations 3rd Edition. Pretince hall.

\section{CITAR ESTE ARTICULO:}

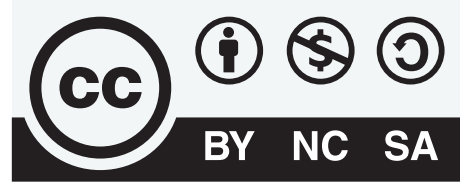

CREATIVE COMMONS RECONOCIMIENTO-NOCOMERCIAL-COMPARTIRIGUAL 4.0.

Ordóñez Espinoza, C. G., Ordóñez Espinoza, M. C., Rodríguez Pillaga, R. T., \& Orbe Guaraca, M. P. (2021). Liderazgo del siglo XXI en las instituciones públicas. RECIMUNDO, 5(1), 164-174. https://doi.org/10.26820/recimundo/5.

(1).enero.2021.164-174 